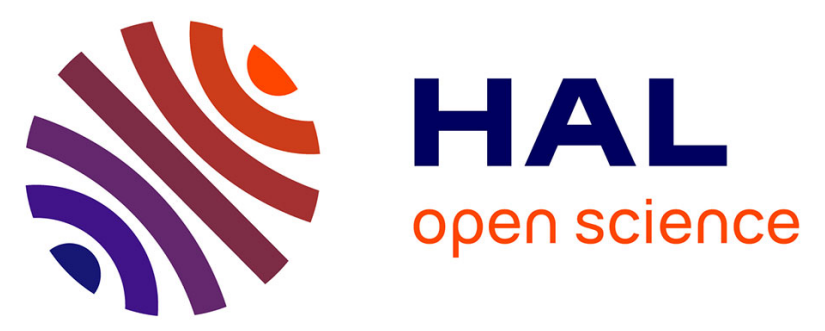

\title{
Twin-scale Vernier micro-pattern for Visual Measurement of 1-D in-plane absolute displacements with increased range-to-resolution ratio.
}

July Andrea Galeano Zea, Patrick Sandoz, Guillaume J. Laurent, Lucas Lopes Lemos, Cédric Clévy

\section{To cite this version:}

July Andrea Galeano Zea, Patrick Sandoz, Guillaume J. Laurent, Lucas Lopes Lemos, Cédric Clévy. Twin-scale Vernier micro-pattern for Visual Measurement of 1-D in-plane absolute displacements with increased range-to-resolution ratio.. International Journal of Optomechatronics, 2013, 7, pp.222-234. 10.1080/15599612.2013.808493 . hal-00869529

\section{HAL Id: hal-00869529 \\ https://hal.science/hal-00869529}

Submitted on 3 Oct 2013

HAL is a multi-disciplinary open access archive for the deposit and dissemination of scientific research documents, whether they are published or not. The documents may come from teaching and research institutions in France or abroad, or from public or private research centers.
L'archive ouverte pluridisciplinaire HAL, est destinée au dépôt et à la diffusion de documents scientifiques de niveau recherche, publiés ou non, émanant des établissements d'enseignement et de recherche français ou étrangers, des laboratoires publics ou privés. 


\title{
Twin-scale Vernier Micro-Pattern for Visual Measurement of 1D in-plane Absolute Displacements with Increased Range-to-Resolution Ratio
}

\author{
July Galeano Zea*§, Patrick Sandoz ${ }^{\dagger}$, Guillaume J. Laurent*, Lucas Lopes Lemos ${ }^{* \ddagger}$ and Cédric Clevy* \\ *AS2M Department, FEMTO-ST Institute, CNRS, ENSMM, Université de Franche-Comté, France \\ Email: guillaume.laurent@ens2m.fr, cedric.clevy@femto-st.fr \\ †Applied Mechanics Department, FEMTO-ST Institute, CNRS, Université de Franche-Comté, France \\ Email: patrick.sandoz@univ-fcomte.fr \\ Fax: +33 (0) 381666700 \\ ${ }^{\ddagger}$ EU4M : EUropean Master in Mechatronics and MicroMechatronics Systems \\ Email: lucas.lopes@eu4m.eu \\ $\S$ Institute of Aviation, Warsaw, Poland \\ Email: july.galeano@ilot.edu.pl
}

\begin{abstract}
This paper presents a visual method for 1D in-plane displacement measurement which combines a resolution of a few nanometers with an unambiguous excursion range of $168 \mu \mathrm{m}$. Furthermore, position retrieval is only based on elementary phase computations and thus might become compatible with high-rate processing by implementing the processing algorithm on high speed computing architectures like a DSP or a FPGA device.

The method is based on a twin scale Vernier micro-pattern fixed on the moving target of interest. The two periodic grids have slightly different periods in order to encode the period order within the phase difference observed between the two sub-patterns. As a result, an unambiguous range of $168 \mu \mathrm{m}$ is obtained from grid periods of $8 \mu \mathrm{m}$ and $8.4 \mu \mathrm{m}$. The resolution is evaluated to be of $11.7 \mathrm{~nm}$ despite remaining mechanical disturbances. Differential measurements demonstrated indeed a measurement accuracy better than $5 \mathrm{~nm}$.
\end{abstract}

\section{INTRODUCTION}

Advances in the field of biology, opto-mechatronics, and micro-nanotechnology, demand at each time the use of highaccuracy position measurement methods for the manipulation of a target at the micro-nano-scale. In most applications, visual methods have played an important role in high accurate position assessment. They allow having a visual evaluation of the changes that occur in a determined field of view (FOV). However for visual methods, the performance of high accurate position measurements over a wide measurement range remains a challenge that is difficult to meet.

The resolution of an imaging system depends on the numerical aperture, the wavelength of the illumination (Rayleigh limit) and the sensor pixel size [1]. This might suggest that motion resolution is limited by the wavelength of light. However, nanometer position resolution is possible. The limitations on position resolution are determined by the resolution and precision of the optics and imager, by substrate vibrations, and by the quantum nature of light. Also, the algorithm used to estimate motion between video images can also limit the position estimation resolution [2]. Among the state of the art sub-pixel motion detection algorithms, phase-like correlation methods are the most widely used. For example, this kind of method has been implemented by Moddemeijer [3] reporting a resolution of $13.3 \mathrm{~nm}$. Also, authors like Douglas [4] and Jun Gao et al. [5], have combined correlation with statistical methods. As a result resolutions around the $10 \mathrm{~nm}$ and $1 \mathrm{~nm}$ respectively were achieved.

The main drawback of these correlation-like methods remains in the useful FOV. Those methods are feature dependent so that salient objects have to remain inside the region of interest to be analyzed limiting thus the allowed range of displacement. To overcome such drawback, a technique based on pseudo-periodic patterns has been proposed by different authors [6], [7], [8], [9]. In these methods, the pseudoperiodic pattern is attached to the mobile object of interest. Then images from the pattern are acquired. In this way, when a displacement occurs, it can be calculated from the pattern images. High-accurate displacements calculations were reported with this approach even in case of displacements that are much wider than the camera's FOV. By comparing the relative motion of a reference grid located on a MEMS substrate with a measurement grid located on the mobile part of the MEMS, Yamahata et al. demonstrated a sub-nanometer resolution in the measurement of 1D displacements [10].

In these periodic grid methods, the high accuracy is obtained from some Fourier-like processing which allows an easy calculation of the position directly from the phase value tied to the grid's periodicity. Nevertheless and up to here, the range of measurement is limited by the grid's period due the phase uncertainty of $2 \pi \mathrm{k}$ given by the arctangent function implied in 


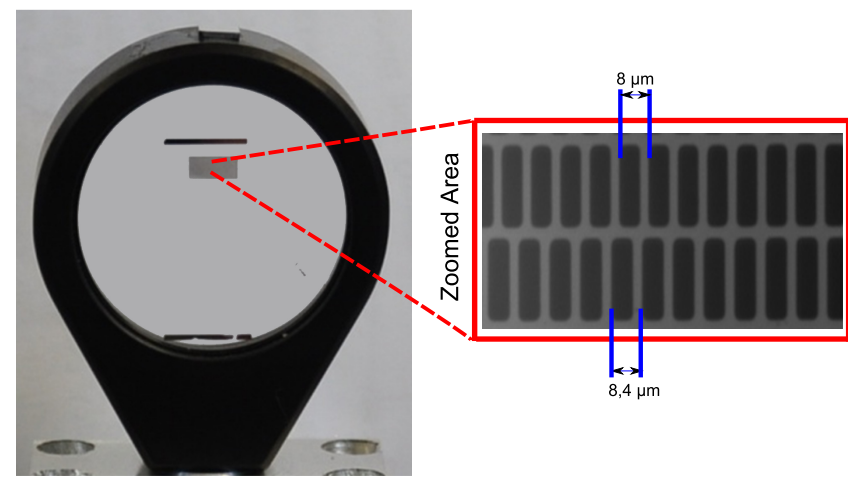

Fig. 1. Glass cover-slip with the periodic-pattern.

phase retrieval as in Yamahata's case [10]. This means that the position measurement is cyclical and can be used to determine the target's position only within a single cycle. To overcome this uncertainty, the information given by the binary code is used. This binary code allows a coarse but absolute determination of the actual target's position allowing measurements larger than the grid's period and camera's FOV. The need to retrieve some binary code is however time-consuming and thus often incompatible with real-time applications.

In this article we present a novel concept in periodicabsolute patterns. It consists of a Vernier-like pattern with a twin-scale periodic grid with two different pitch sizes $\lambda_{1}$ and $\lambda_{2}$ respectively. This configuration is well known as a "Two-Wavelength Method" (TWM) commonly used in holography and interferometry applications [11], [12]. Equivalent to Galeano et al. [8], high accurate position is obtained from phase evaluation. Nevertheless in TWM, phase ambiguities are not removed by means of a binary code but through the difference between the phase values obtained from both ruling grids. The unambiguous range is thus extended as defined by:

$$
\lambda_{T}=\frac{\lambda_{1} \lambda_{2}}{\left|\lambda_{1}-\lambda_{2}\right|}
$$

For instance, if $\lambda_{1}$ and $\lambda_{2}$ equal to $8 \mu \mathrm{m}$ and $8.4 \mu \mathrm{m}$ respectively, thus $\lambda_{T}=168 \mu \mathrm{m}$. In this way, since no binary decoding is necessary, the present method is suitable for real time application.

These grid methods can be seen as derived from the wellknown Vernier principle since the moving periodic pattern attached to the target acts as the secondary Vernier scale in the Vernier caliper with respect to the static primary scale that is formed in vision by the pixel frame of the digital camera. Section II of this article describes the principle and the image processing of the proposed method.

Section III presents the performance of the method in terms of repeatability, accuracy and resolution. Finally, conclusions and prospects are discussed in section IV.

\section{Materials And Methods}

Figure1 presents the periodic pattern used in this article. Photolithography processes were used to imprint the periodic pattern onto the substrate. Periodic strips are made of an aluminum layer deposited onto a transparent glass coverslip $(120 \mu \mathrm{m}$ thick). The grid periods of $8 \mu \mathrm{m}$ and $8.4 \mu \mathrm{m}$ respectively were chosen in order to be compatible with low magnification lenses (typically $10 \times$ or $20 \times$ ) thus keeping a comfortable working distance in practice.

Then this pattern is attached to the target of interest in order to be observed by the vision system. Figure 2 presents an image of the periodic pattern as recorded experimentally using a $20 \times$ microscope lens. It is composed of a series of ruling grids where the $8 \mu \mathrm{m}$ and $8.4 \mu \mathrm{m}$ grids are distributed alternatively.

Previous to any displacement assessment, the calibration of the imaging system can be carried out by means of the size references provided by the known grids periods. This implies to know: first the exact position at which we find the grids with pitch of $8 \mu \mathrm{m}$ and $8.4 \mu \mathrm{m}$, and second the number of pixels equivalent to one grid period in the recorded image. The localization of the grids in the acquired images is based on classical image processing methods involving thresholding and center of gravity determination. The image raws centered on each $8 \mu \mathrm{m}$ or $8.4 \mu \mathrm{m}$ grids are thus determined. The determination of the grid periods in pixels is based on Fourier transform with subsequent phase adjustments in order to get an accurate evaluation of these processing parameters.

In Figure 2 the red lines define a working interval of 100 lines used as an input data for image processing for position assessment. All lines in this set carry the same spectral information but each one with an independent pattern of noise due to photolithography imperfections and electronic noise. Then, from this set, by summing 20 lines per pattern, we obtain a noise-averaged signal as presented in Figure 3. This signal is used as the input data which is processed in two steps: a first one concerning high accurate positioning and a second one concerning coarse-absolute position evaluation.

\section{A. Phase Calculation}

The high accurate position is calculated from the phase value obtained by Discrete Fourier Transform (DFT). At this stage, we perform two specific adjustments in the DFT:

- in order to save time and to achieve real-time processing, we compute a single spectral component instead of performing a complete DFT.

- to avoid disturbances due to the limited extension of the line pattern, we choose to perform a windowed Fourier transform, using the analysis function expressed by equation 2 and represented in Figure 4:

$$
a(x)=W\left(x-x_{0}\right) e^{(-2 i \pi x / T)}
$$

with $W\left(x-x_{0}\right)$ the Gaussian envelope calculated at the central pixel $x_{0}$, and $T$ the period of the considered line pattern in the recorded image as previously estimated.

The Fourier transform is then calculated as indicated by equation 3 : 


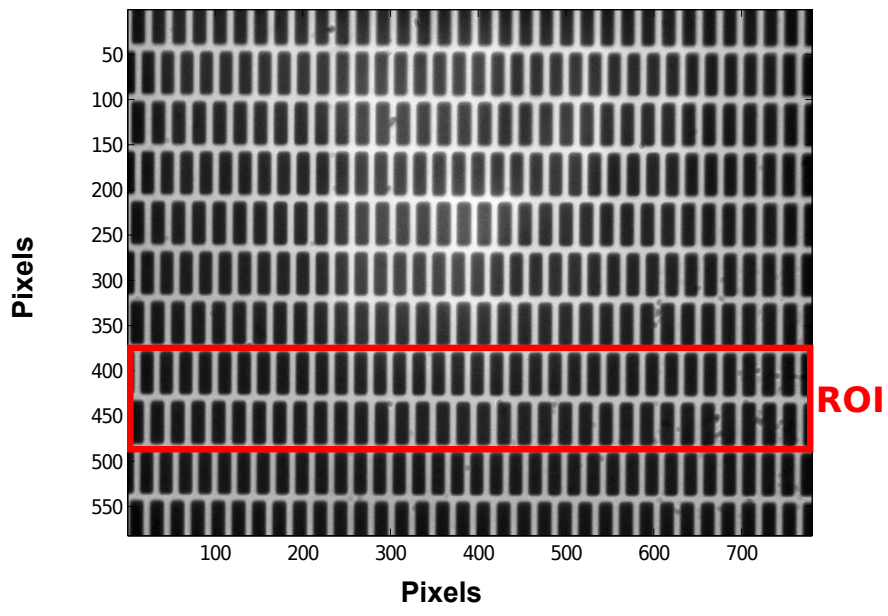

Fig. 2. Recorded image of the periodic pattern stuck on the object of interest. Red lines define the region of interest used for processing.

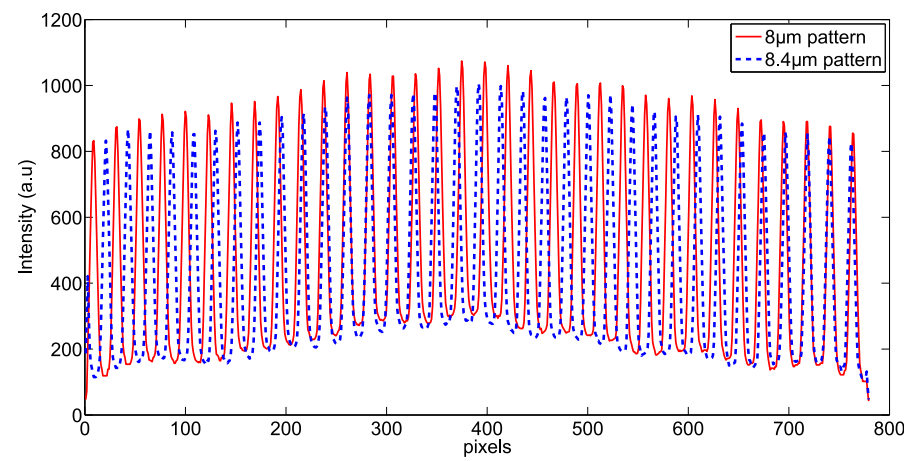

Fig. 3. Intensity profile obtained after summing 20 lines for each periodic sub-pattern (red: $8 \mu \mathrm{m}$, dashed blue: $8.4 \mu \mathrm{m}$ ).

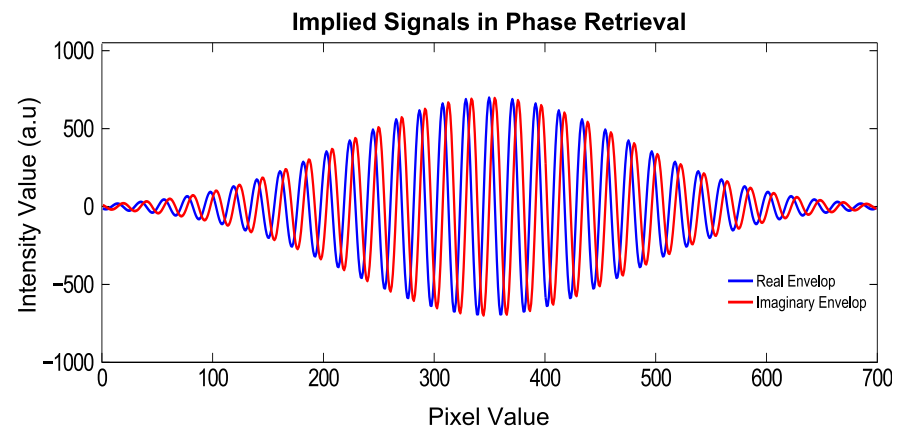

Fig. 4. Fourier phase analysis function; blue: real part, red: imaginary part.

$$
S(f)=\sum s(x) \cdot a(x)
$$

where $s(x)$ is the sum of the twenty lines of one grid. Once the transform $T$ is calculated, the phase $\phi$ is obtained using the arctangent function, as given by the expression 4 :

$$
\phi(f)=\arg S(f)=\arctan 2(\operatorname{Im} S(f), \operatorname{Re} S(f))
$$

The signal used for phase calculation is periodical but not sinusoidal. Considered in the frequency domain, the analysis function behaves as a spectral filter whose shape is given by its Fourier transform. The wider the analysis function in the space (image) domain, the narrower the spectral filter. The choice of a wide analysis function (cf. Figure 4) ensures that only frequencies very close to the pattern frequency may distort the phase computation. In particular signal non-linearities which correspond to harmonics of the signal frequency in the spectral domain - are filtered out by such a wide analysis function [13]. The only effect of signal non-linearities is thus a reduction of the magnitude of the fundamental spectral lobe and this can be seen as a simple decrease of the signal to noise ratio.

This point makes our phase computation approach different from phase shifting techniques commonly used in interferometry. In the latter, the choice of a constant phase shift - usually pi/2 - is responsible for measurement errors at twice the fringe frequency and due to signal detection non-linearities. Our case differs in two main points: Firstly we consider more samples per fringe (more than 10 instead of 4). Secondly the equivalent phase shift is not a perfect fraction of fringe and this avoids a coherent accumulation of errors over consecutive fringes.

The mentioned procedure in applied twice for the line sets corresponding to periods $\lambda_{1}$ and $\lambda_{2}$ respectively. In this way, two phases $\phi_{1}$ and $\phi_{2}$ are obtained, both with a $2 k \pi$ ambiguity, as in the example presented in Figure 5a.

\section{B. Absolute positioning}

High accurate absolute position measurement is defined in a range defined by equation 1 . The high accuracy is defined by the smallest period grid $\lambda_{1}$, while the absolute value is obtained from the phase difference between both grids:

$$
\phi_{T}=\phi_{1}-\phi_{2},
$$

where $\phi_{1}$ and $\phi_{2}$ are the phases measured for $\lambda_{1}$ and $\lambda_{2}$ respectively. The phase difference $\phi_{T}$, corresponding to the phase value for a grid with period $\lambda_{T}$, renders the exact number of $\lambda_{1}$ cycles in the range defined by $\lambda_{T}$. This difference will give us the absolute coarse position. This absolute position compensates the ambiguities of $2 k \pi$ obtained from the high accurate position from $\phi_{1}$. As presented in the example of Figure $5 \mathrm{~b}$, the total phase difference $\phi_{T}$ gives the number of $2 \pi$ terms to add to the accurate phase value $\phi_{1}$. 
This adjustment can be determined by direct point-to-point comparison of the value $\phi_{T}$ to $\phi_{1}$ as expressed by equation 6:

$$
\phi_{a b s}=\phi_{1}+2 \pi . \operatorname{int}\left[\frac{\phi_{T} \cdot N}{2 \pi}\right],
$$

where int corresponds to the function returning the integer the closest of the input argument, and $N$ is the total number of entire periodical cycles of the phase $\phi_{1}$ defined in a range $\lambda_{T}$ $\left(N=\lambda_{T} / \lambda_{1}\right)$. After phase compensation of $\phi_{a b s}$, the absolute high accurate position is obtained. In the given example, the final result is presented in $5 \mathrm{c}$.

\section{EXPERIMENTAL RESULTS AND PERFORMANCES}

\section{A. Method used for system Characterization}

A series of experiments were performed in order to evaluate the method's capabilities in terms of repeatability, resolution and absolute positioning. The set-up used in these experiments is presented in Figure 6. As can be seen, the vision system used in this work is composed of a red light-emitting-diode (LED) (Luxeon Star:0906LXHLND98), a microscope objective (Edmund Optics Din $20 \times$, N.A. 0.4), and a CCD camera (Allied Vision Technology Guppy F-046, $780 \times 582$ pixels, cell size $8.3 \mu \mathrm{m})$ connected to a computer. Images of the periodic pattern incident on the CCD sensor are recorded in the computer's memory. In this way, 1D in-plane displacements of the target can be determined directly from the images of the periodic pattern. A servocontrolled piezoelectric transducer (PZT) is used as a calibrated moving target. This motor is equipped with a high accurate capacitive sensor which is used as a target position reference for visual measurement evaluation.

In practice, the pattern mask was always attached to the PZT, illuminated in transmission with the red light emitting diode and imaged on the CCD camera through the $20 \times$ magnification microscope objective. The intervals at which the images were taken depended on the kind of experiment to perform. These experiments and their corresponding results are explained in details in the following subsections.

\section{B. Evaluation of the reproducibility}

The system reproducibility was measured by maintaining the PZT static (switched off) and taking images of the pattern at a rate of $1 \mathrm{fps}$. Retrieved position values are plotted in Figure 7. The peak-valley deviation is $27 \mathrm{~nm}$ while the standard deviation is $3.9 \mathrm{~nm}$. With these results, we estimate the system reproducibility to be $11.7 \mathrm{~nm}(3 \sigma)$. This reproducibility is consistent with the theoretical resolution of Fourier grid methods of $p / N$ with $p$ the grid's period and $N$ the total image pixels [14].

As our approach consists in a discrete windowed Fourier transform, the intrinsic limits to phase resolution are only given by the signal to noise ratio and the extension of both the analysis function and the recorded pattern image. With the configuration used in our experiments, the 8-bits camera is the most limitative factor to the achievable position resolution.
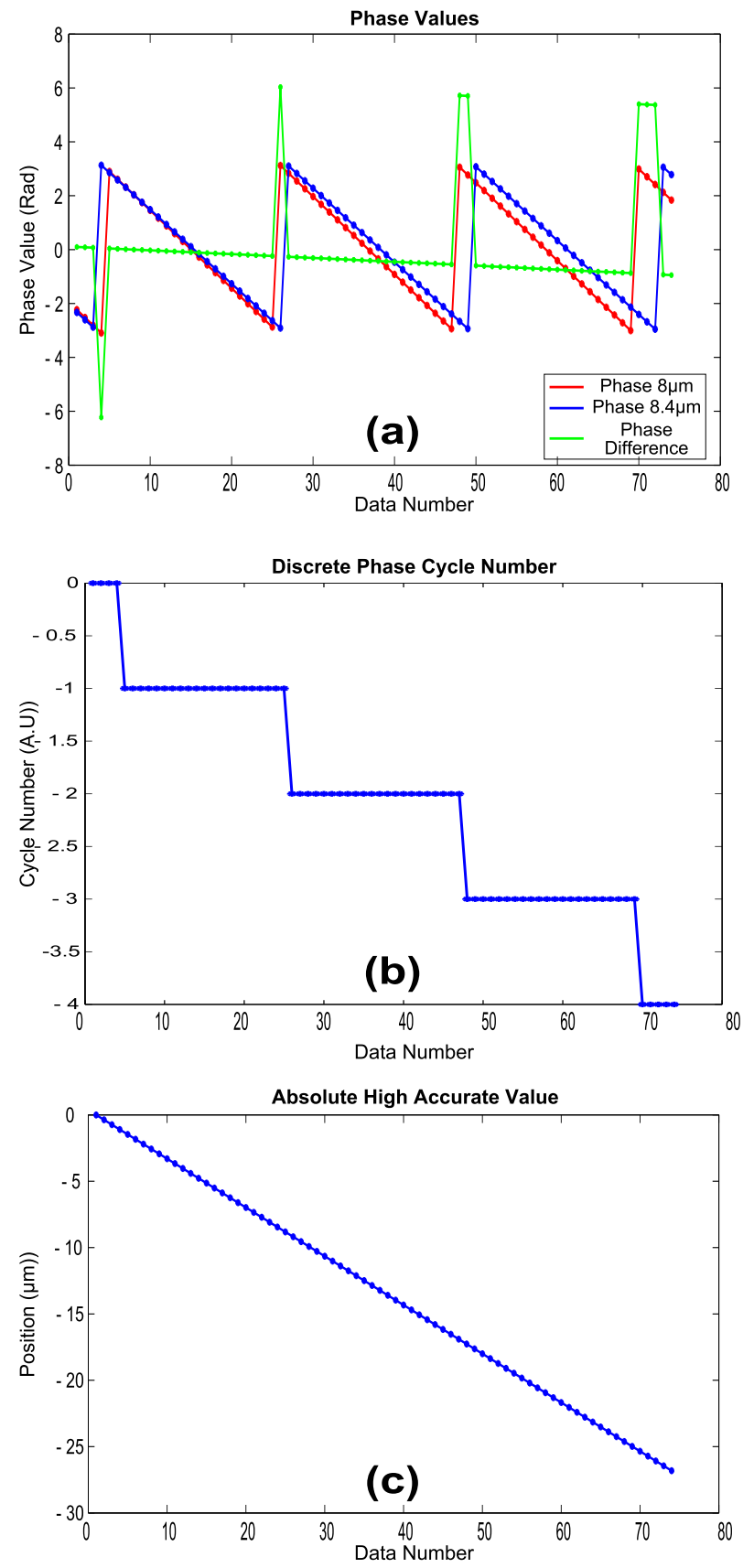

Fig. 5. Example of the phase values obtained when the periodic pattern (presented in Figure 2) is shifted by $27 \mu \mathrm{m}$. a). Phase value obtained at 8 and $8.4 \mu \mathrm{m}$ grids, together with their phase difference $\phi_{T}$. Due to the $2 k \pi$ uncertainty, phase unwrapping has to be performed on $\phi_{T}$. b). Discrete phase cycle number obtained from the phase $\phi_{T}$. c). Final result: high-accurate absolute position measurement. 


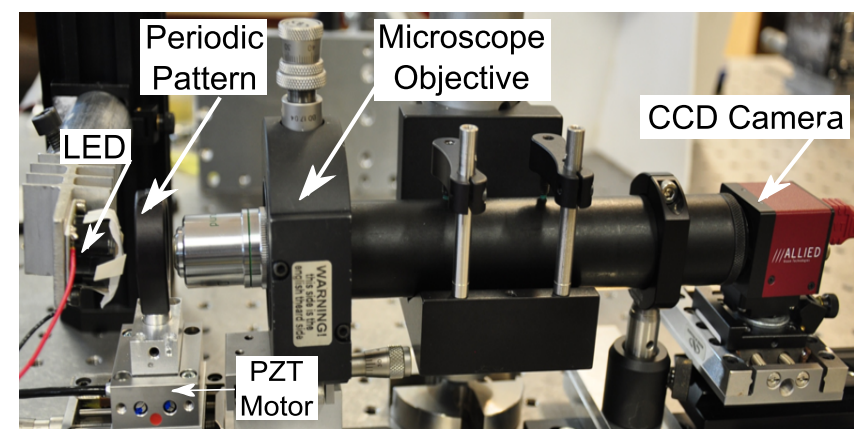

Fig. 6. Experimental set-up used in high-accurate position assessment.

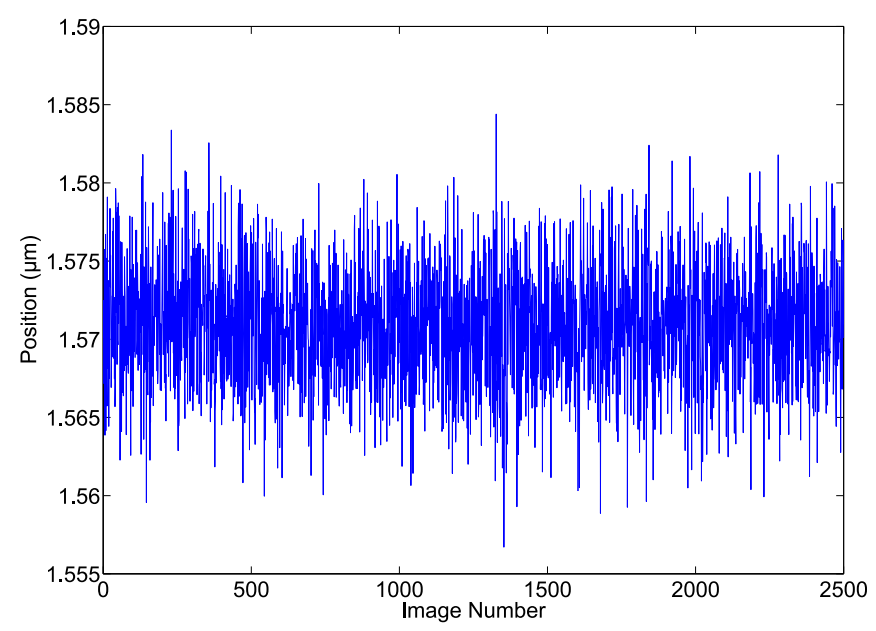

Fig. 7. Evolution of the retrieved position values while the PZT is static.

The environment sources of disturbances and the setup lack of stiffness is another limitative factor in our experiments but this condition in not intrinsic to the measurement method explored in this paper. Thus, better results are expected in the future. Yamahata did indeed demonstrate a subnanometer resolution by this kind of approach by removing rigid-body disturbances thanks to a static reference pattern [10].

\section{Accuracy in position measurement}

In order to evaluate the method's accuracy, a short range but high accurate PZT motor (P-753.1CD from Physics Instruments) was used. The positions rendered by the internal capacitive sensor of this motor were used as the reference values. This motor has a theoretical resolution close to $0.05 \mathrm{~nm}$ in close-loop operation, and a repeatability of $1 \mathrm{~nm}$.

Displacements by steps of $12 \mathrm{~nm}$ were performed with this motor over a range of $800 \mathrm{~nm}$. For each step, one image was acquired together with the position given by the internal motor's capacitive sensor. An interval of time of $1 \mathrm{sec}$ was used between the motors' positioning and data acquisition in order to wait for the motor stability. The results of this experiment are depicted in Figure 8a. Figure $8 \mathrm{~b}$ presents the difference between the measurement obtained from the
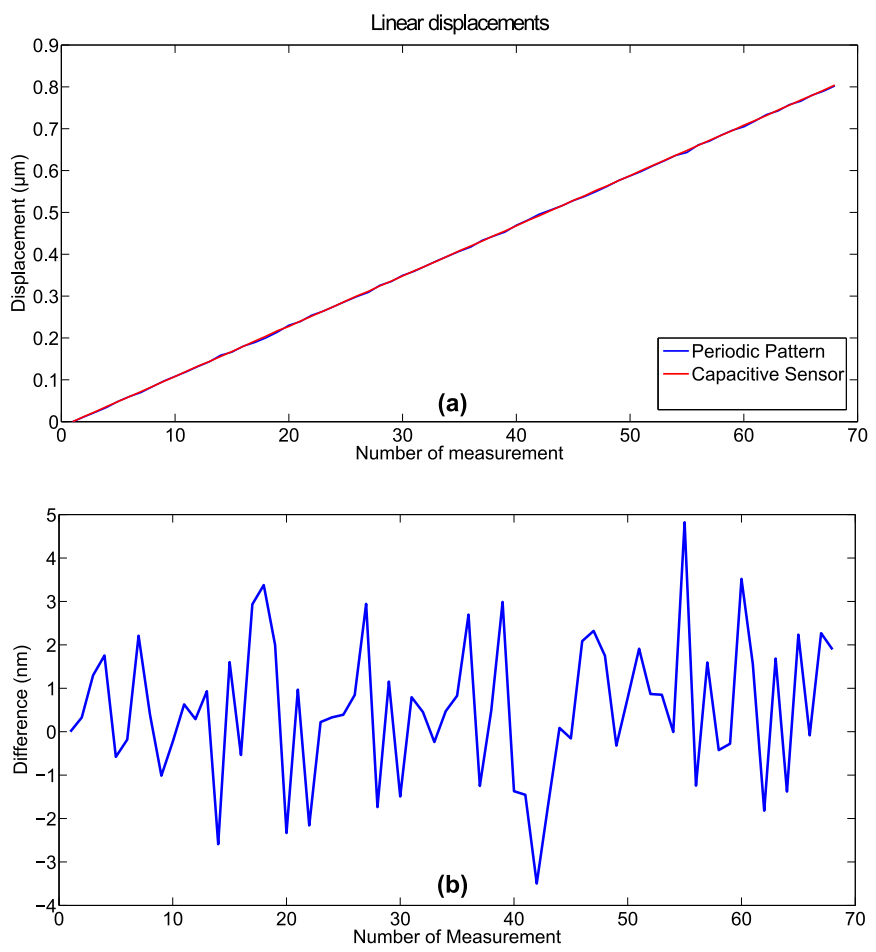

Fig. 8. Comparison of position values given by the PZT capacitive sensor and the periodic-pattern image processing. Steps of $12 \mathrm{~nm}$ were applied over an excursion range of $800 \mathrm{~nm}$ : a) positions returned by the capacitive sensor and by vision, b) position differences.

capacitive sensor and the periodic grid. It can be seen a maximum of $4.8 \mathrm{~nm}$ in position assessment difference. The standard deviation of this difference is $1.6 \mathrm{~nm}$, which is a good indicator of the method's accuracy bearing in mind that the commanded positions are at the limit of the system reproducibility. The mismatch thus observed between the estimated reproducibility and accuracy is attributed to the different durations of the experiments. The evaluation of the reproducibility was carried out for a long period of 41 minutes, when short range tests were done within only 70 seconds. The thermal drift and the other slow disturbances are less visible on shorter tests.

\section{Test of Absolute Positioning}

Finally using a wider range PZT motor (P-541.2CD from $\mathrm{PI}$ ), random displacements were performed in order to evaluate the system's capabilities in absolute positioning. This motor has a theoretical resolution close to $0.3 \mathrm{~nm}$ in close-loop operation. Its repeatability is around $5 \mathrm{~nm}$ and has a linearity of $0.03 \%$ with an excursion range of $100 \mu \mathrm{m}$. With this motor, a series of random steps were performed within a range of $100 \mu \mathrm{m}$ (full PZT range). Then, for each step, a series of 50 images were taken at a rate of $1 \mathrm{fps}$. At the same time the position from the internal PZT capacitive sensor was recorded. Figure 9 presents the results using this protocol. Figure 9.a depicts the position measurements using the high accurate- 
relative and absolute-coarse data. It can be seen that the accurate position measurement values (in blue line) remains between 0 and $8 \mu \mathrm{m}$ due to the phase value uncertainty of $2 k \pi \mathrm{rad}$. In red line is presented the coarse absolute position, which is obtained by multiplying the phase difference by a factor of $\lambda_{T} / \pi$. Although this first result corresponds to a coarse positioning, it offers a first trace of the target position which is very close to the displacement set-points. Nevertheless, this absolute-coarse positioning is highly noisy. As can be see in Figure 9.b the difference between coarseabsolute and capacitive sensor positions are of the order of $500 \mathrm{~nm}$. Due to this fact, it is necessary to use the information provided by the high-accurate relative measurement. At this stage, the value obtained from the $8 \mu \mathrm{m}$ grid is compensated using the information from the absolute coarse position using equation 6. Figure 9.c presents the difference between the measurements obtained by the method and the ones obtained by the capacitive sensor. Since these positions are composed of a series of forward and backward routines, the quality of the method's measurements are sensitive to the motor's repeatability, linearity and mechanical set-up stability. However, the maximum position difference achieved is around $100 \mathrm{~nm}$ and the average of the standard deviation obtained at each position step corresponds to $2.7 \mathrm{~nm}$.

\section{CONCLUSION}

The application of the Two-Wavelength Method (TWM) for the measurement of 1D in-plane displacement by vision has been successfully demonstrated in this paper and a resolution of $11.7 \mathrm{~nm}$ was obtained. Because of remaining mechanical disturbances, better performances can still be expected as confirmed by the method accuracy estimation of $5 \mathrm{~nm}$ following a protocol in which rigid-body setup disturbances are compensated for.

This level of performance is achieved with an unambiguous measurement range of $168 \mu \mathrm{m}$, leading to an allowed dynamics of more than $10^{4}$ resolved points. This dynamics could still be extended in both directions. On the one hand, the resolution can be improved by choosing shorter grid periods; the limit will be given by diffraction to around $1 \mu \mathrm{m}$ in visible light. On the other hand, the unambiguous range can be extended by inserting a third grid (or even more) in the Vernier micropattern with a different period in order remove the $2 k \pi$ phase jumps of the equivalent wavelength $\lambda_{T}$ and compensate for them. As a Vernier rule, the shorter the distance between the different periods, the wider the equivalent wavelength is; but the most sensitive is the phase difference with respect to the level of noise. A trade-off has thus to be found in practice between the width of the unambiguous range and the detection efficiency as a function of the vision system noise figure.

This approach involves only elementary phase calculations and thus the image processing algorithm could be implemented within a high-speed computation unit as a DSP or under a FPGA architecture as proposed by [15]. In such a prospect the measurement rate would be mainly limited by the frame
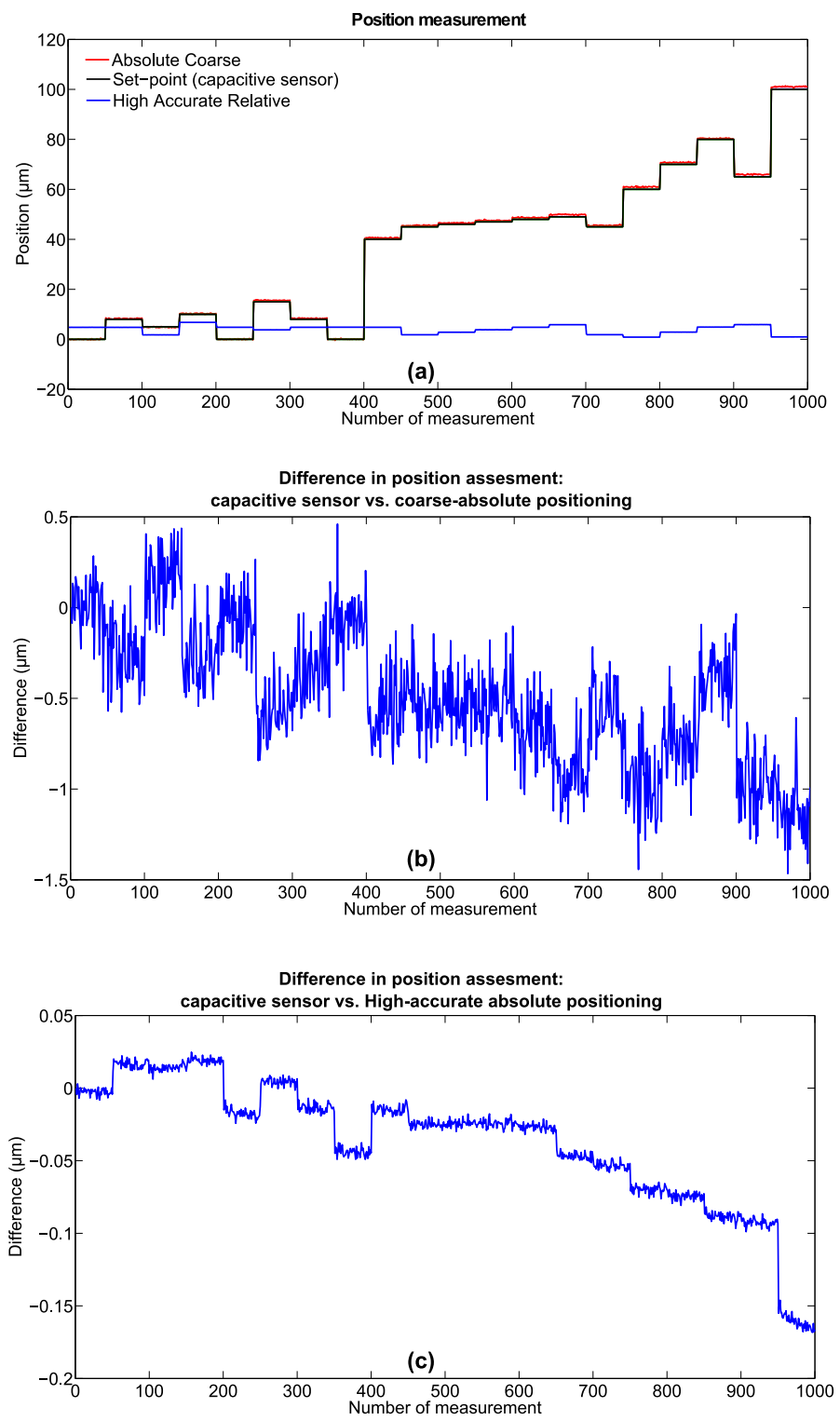

Fig. 9. Random positioning: a). Position given by the coarse and highaccurate-relative measurements, b). Difference between absolute-coarse position and capacitive sensor measurements, c). Difference between Highaccurate absolute position and capacitive sensor measurement.

rate allowed by the camera for a region of interest limited to two sets of several image raws.

\section{ACKNOWLEDGMENT}

We acknowledge the Region of Franche-Comté and OSEO for project funding. We thank David Guibert for assistance in mechanical designs. This work is supported by the national ANR-Emergence MYMESYS-project (ANR-11-EMMA-006: High Performances Embedded Measurement Systems for multiDegrees of Freedom Microsystems), the LABEX ACTION, and the French RENATECH network and its FEMTO-ST technological facility. 


\section{REFERENCES}

[1] R. Vollmerhausen and R. Driggers, Analysis of sampled imaging systems. Spie Press, 2000.

[2] C. Davis and D. Freeman, "Using a light microscope to measure motions with nanometer accuracy," Optical Engineering, vol. 37, p. 1299, 1998.

[3] R. Moddemeijer, "On the determination of the position of extrema of sampled correlators," Signal Processing, IEEE Transactions on, vol. 39, no. 1, pp. 216-219, 1991.

[4] S. Douglas, "Frequency-domain subpixel position estimation algorithm for overlay measurement," in Proceedings of SPIE, vol. 1926, 1993.

[5] J. Gao, C. Picciotto, W. Wu, and W. Tong, "From nanoscale displacement sensing and estimation to nanoscale alignment," Journal of Vacuum Science \& Technology B: Microelectronics and Nanometer Structures, vol. 24, p. 3094, 2006.

[6] P. Masa, E. Franzi, and C. Urban, "Nanometric resolution absolute position encoders." CSEM Scientific and Technical Report, 2008.

[7] D. Boyton, "Position encoder using statistically biased pseudorandom sequence," Apr. 18 2003, uS Patent App. 10/399, 470.

[8] J. Galeano-Zea, P. Sandoz, E. Gaiffe, J. Prétet, and C. Mougin, "PseudoPeriodic Encryption of Extended 2-D Surfaces for High Accurate Recovery of any Random Zone by Vision," International Journal of Optomechatronics, vol. 4, no. 1, pp. 65-82, 2010.

[9] P. Sandoz, R. Zeggari, L. Froehly, J. Prétet, and C. Mougin, "Position referencing in optical microscopy thanks to sample holders with outof-focus encoded patterns," Journal of Microscopy, vol. 225, no. 3, pp 293-303, 2007.

[10] C. Yamahata, E. Sarajlic, G. Krijnen, and M. Gijs, "Subnanometer translation of microelectromechanical systems measured by discrete fourier analysis of ccd images," Microelectromechanical Systems, Journal of, vol. 19, no. 5, pp. 1273-1275, 2010.

[11] K. Creath, "Step height measurement using two-wavelength phaseshifting interferometry," Applied optics, vol. 26, no. 14, pp. 2810-2816, 1987.

[12] P. de Groot, "Extending the unambiguous range of two-color interferometers," Applied optics, vol. 33, no. 25, pp. 5948-5953, 1994.

[13] K. Creath, "Phase-measurement interferometry techniques," Progress in optics, Elsevier, vol. 26, pp. 349-393, 1988.

[14] B. Zhao and A. Asundi, "Microscopic grid methods resolution and sensitivity," Optics and lasers in engineering, vol. 36, no. 5, pp. 437450, 2001.

[15] S. Strube, G. Molnar, and H.-U. Danzebrink, "Compact field programmable gate array FPGA-based multi-axial interferometer for simultaneous tilt and distance measurement in the sub-nanometre range," Measurement Science and Technology, vol. 22, no. 9, 2011. 